\title{
Thoracic endovascular aortic repair for recurrent stroke due to atheromatic plaque in the proximal descending aorta: a case report
}

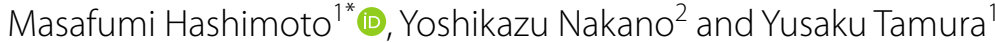

\begin{abstract}
Background: Diastolic retrograde flow in the descending aorta (DAo) may occur in the presence of atherosclerosis and may be overlooked as a mechanism of retrograde embolization in patients with stroke. We performed thoracic endovascular aortic repair (TEVAR) in a patient with recurrent cerebral infarctions for treatment of aortic aneurysm with atheromatic plaque, which was considered as the source of embolism.
\end{abstract}

Case presentation: A 56-year-old man with a history of idiopathic thrombocytopenia and hypertension was referred to our hospital with paralysis of the right upper and lower limbs. Multiple cerebral infarctions were found and treated; however, 1 month later, another cerebral infarction developed. A small saccular aortic aneurysm with plaque was found beyond the left subclavian artery, and this site was deemed as the source of embolism. We performed TEVAR to prevent further recurrence of cerebral infarctions. No cerebral infarctions were observed 6 months post-operation.

Conclusions: TEVAR is a useful treatment for not only aortic aneurysm and dissection, but also cerebral infarctions caused by an embolic source proximal to the DAo due to retrograde aortic blood flow.

Keywords: Atheromatic plaque, Idiopathic thrombocytopenia, Retrograde aortic flow, Stroke, Thoracic endovascular aortic repair

\section{Background}

An increasing body of evidence suggests that diastolic retrograde flow in the descending aorta (DAo) may frequently occur in the presence of atherosclerosis and become an overlooked mechanism of retrograde embolization in patients with stroke $[1,2]$. Retrograde flow from complex DAo plaques are frequently detected in both determined and cryptogenic stroke and could explain the cause of embolism in all brain territories [1]. We performed thoracic endovascular aortic repair (TEVAR) for recurrent cerebral infarctions due to a distal arch aortic

\footnotetext{
*Correspondence: m.hashimoto0610@gmail.com

${ }^{1}$ Division of Cardiovascular Surgery, Chibaken Saiseikai Narashino Hospital, Social Welfare Organization Saiseikai, Imperial Gift Foundation Inc., 1-1-1 Izumi chou Narashino city, Chiba 275-8580, Japan Full list of author information is available at the end of the article
}

aneurysm with atheromatic plaque, which was considered as the source of embolism.

\section{Case presentation}

A 56-year-old man with a history of idiopathic thrombocytopenia (ITP) and hypertension was referred to our hospital with paralysis of his right upper and lower limbs. The patient had no history of obvious arrhythmias, such as atrial fibrillation. Brain magnetic resonance imaging (MRI) showed multiple cerebral infarctions (Fig. 1a). One month later, while determining the source of the embolus, the patient developed another cerebral infarction (Fig. 1b). Blood examination revealed only $1.1 \times 10^{4} / \mu \mathrm{L}$ platelets $\left(2.7 \times 10^{4} / \mu \mathrm{L}\right.$ using citrate blood sampling) because of ITP; therefore, antiplatelet therapy could not be administered. There were no arrhythmias (including atrial fibrillation), and transthoracic echocardiography showed no obvious intracardiac thrombus. 


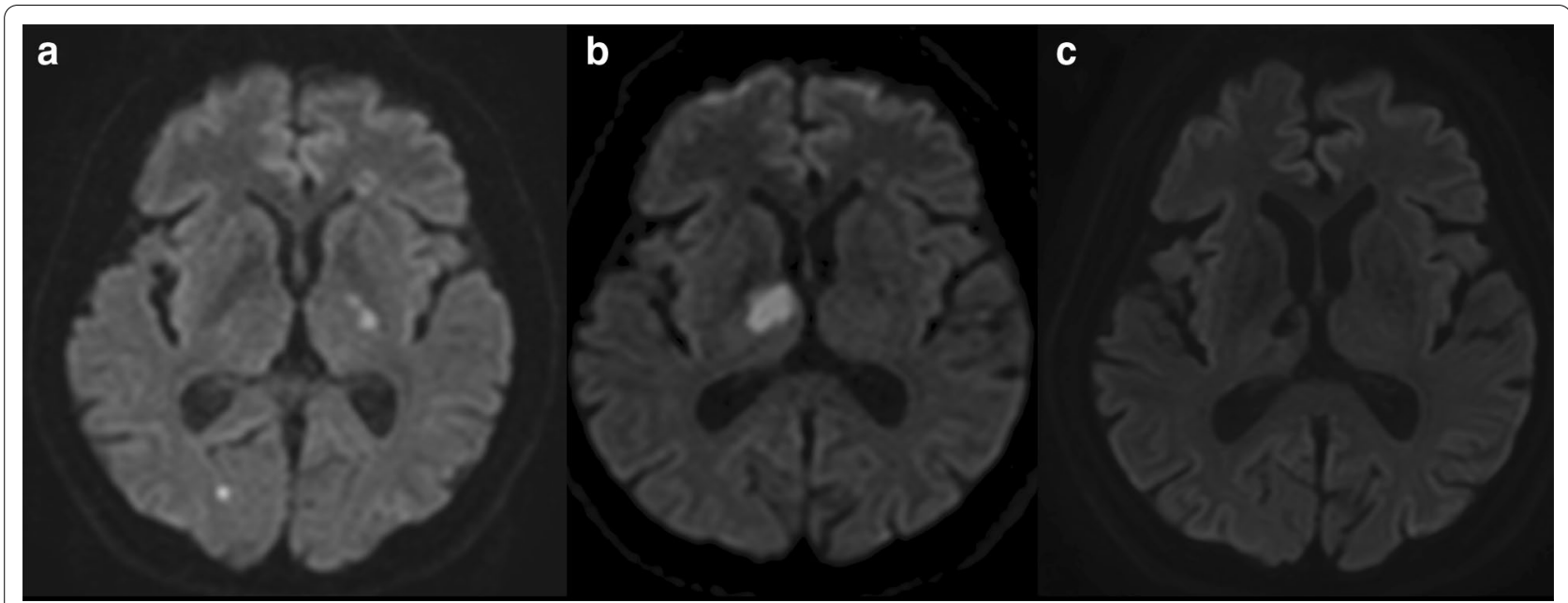

Fig. 1 Brain diffusion-weighted magnetic resonance images. a multiple cerebral infarctions (right occipital lobe, left internal capsule putamen). b Cerebral infarction recurrence (internal capsule of the right thalamus) seen 1 month later. c No cerebral infarction 6 months postoperatively

Transesophageal echocardiography also found no obvious embolic source structure, patent foramen ovale, or decrease in the blood flow of the left atrial appendage. The bubble test results were also negative.

Computed tomography angiography (CTA) and MRI revealed a small saccular DAo aortic aneurysm with atheromatic plaque beyond the left subclavian artery (Fig. 2a, b, and Fig. 3a). No other visible embolic source was found. Thus, the DAo site was considered the source of embolism. TEVAR was planned to prevent stroke recurrence. Preoperatively, immunoglobulin was administered at $30 \mathrm{~g} /$ day for 4 days, and the platelet count improved to $22.6 \times 10^{4} / \mu \mathrm{L}\left(24.0 \times 10^{4} / \mu \mathrm{L}\right.$ using citrate blood sampling).

TEVAR was performed in zone 3 using a Relay Plus (Terumo Corporation, Tokyo, Japan; AU-3414530). Endoleaks were not observed during intraoperative

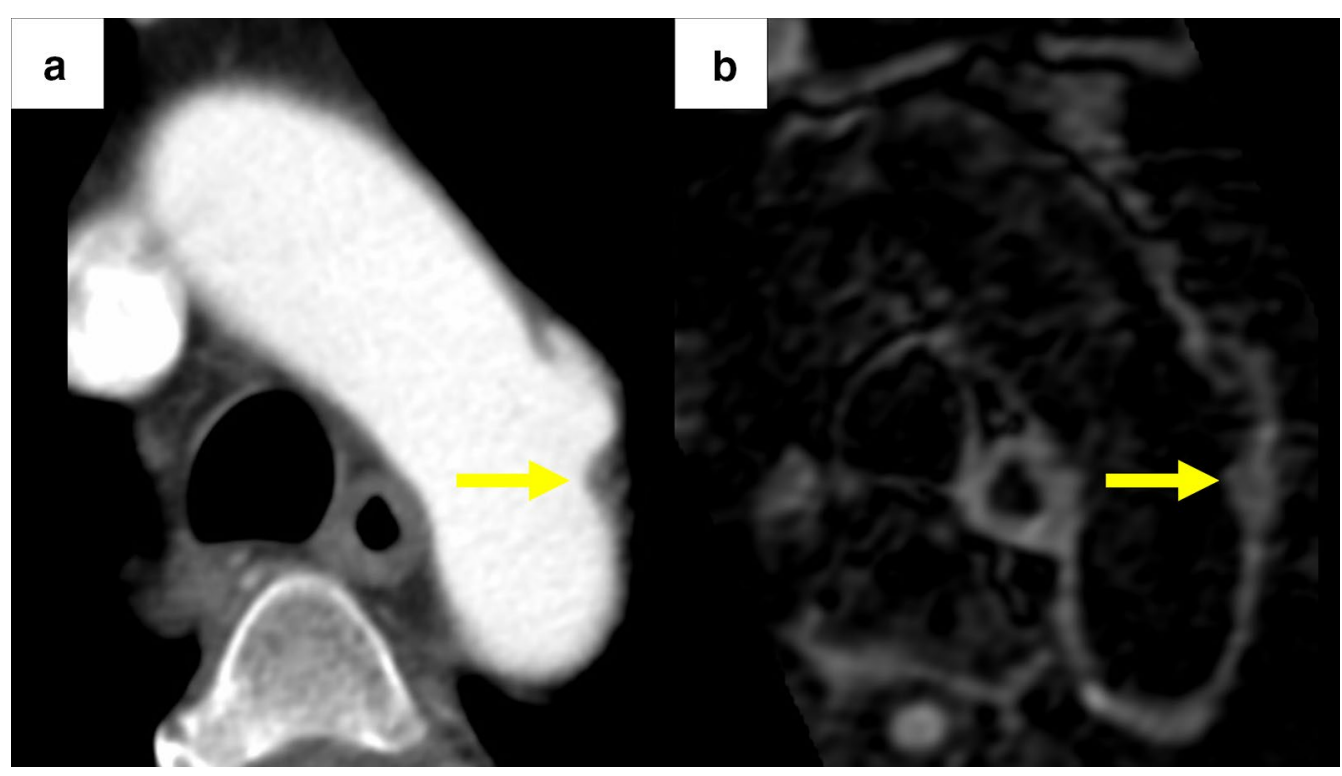

Fig. 2 There is a small saccular aortic aneurysm on the distal arch with the atheromatic plaque. The saccular aneurysm was approximately $35 \mathrm{~mm}$ in diameter and the atheromatic plaque thickness was approximately $4 \mathrm{~mm}$. a Computed tomography angiogram images and $\mathbf{b}$ magnetic resonance images 


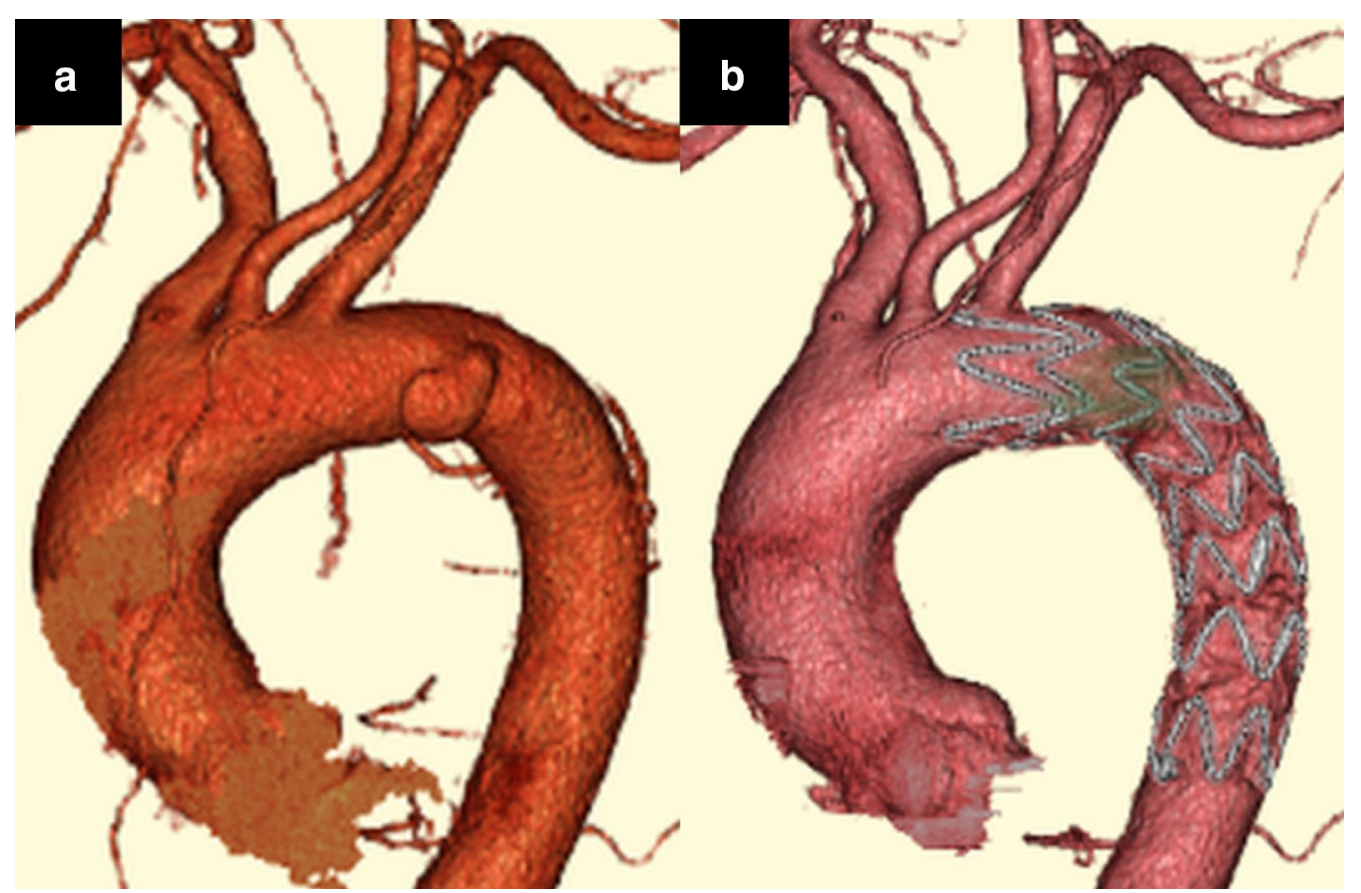

Fig. 3 Computed tomography angiogram images. a Thoracic aortic aneurysm $(35 \mathrm{~mm})$ approximately $15 \mathrm{~mm}$ from the left subclavian artery with atheromatic plaque (preoperative). b Postoperative confirmation of absence of endoleaks

imaging; this was confirmed postoperatively using CTA (Fig. 3b).

After the operation, the motor function recovered, and the patient could walk independently, but the attention disorder and memory impairment persisted. Therefore, the patient was transferred to another hospital for rehabilitation. Six months after the operation, no cerebral infarctions were observed (Fig. 1c).

\section{Conclusions}

Until recently, flow reversal in the thoracic aorta was believed to be a sign of aortic regurgitation. However, studies using transesophageal echocardiography or 4D-MRI showed aortic flow reversal without aortic regurgitation [1,3,4]. Evidence has indicated that arterial stiffness and wall thickness, which are associated with aging and atherosclerosis, can lead to flow reversal due to a mismatch between peripheral and central arterial stiffness $[3,4]$. Typically, retrograde blood flow originates in the first $20-30 \mathrm{~mm}$ of the proximal DAo and can reach all brain-supplying arteries [2]. Thus, atheromas at this site may cause embolic stroke in any brain territory [2].

Since the infarct site was different between the first and recurrent strokes, there was a high possibility of embolic cerebral infarction. Full-body examination did not reveal the source of embolism other than the atheromatic plaque, which was located approximately $15 \mathrm{~mm}$ from the left subclavian artery on the proximal side. If $4 \mathrm{D}-\mathrm{MRI}$ could be performed, it would be clearer that the cause of the stroke was the atheromatic plaque, but unfortunately it was not possible at our institution. Despite the small size of the aneurysm, TEVAR was performed because of the history of ITP and the patient's ineligibility to undergo antiplatelet therapy.

TEVAR is a useful treatment for cerebral infarction caused by an embolic source proximal to the DAo due to retrograde aortic blood flow. We hope that this case report will help in the treatment of cryptogenic stroke.

\section{Abbreviations}

Dao: Descending aorta; CTA: Computed tomography angiography; MRI: Magnetic resonance imaging; TEVAR: Thoracic endovascular aortic repair; ITP: Idiopathic thrombocytopenia.

\section{Acknowledgements}

None declared.

\section{Authors' contributions}

All authors read and approved the final manuscript.

\section{Funding}

None declared

\section{Declarations}

Consent for publication

The patient provided informed consent for the publication of this case report. 


\section{Competing interests}

The authors declare that they have no competing interests.

\section{Author details}

${ }^{1}$ Division of Cardiovascular Surgery, Chibaken Saiseikai Narashino Hospital, Social Welfare Organization Saiseikai, Imperial Gift Foundation Inc., 1-1-1 Izumi chou Narashino city, Chiba 275-8580, Japan. ${ }^{2}$ Division of Neurology Chibaken Saiseikai Narashino Hospital, Social Welfare Organization Saiseikai, Imperial Gift Foundation Inc., Chiba, Japan.

Received: 9 February 2021 Accepted: 18 April 2021

Published online: 28 April 2021

\section{References}

1. Harloff A, Simon J, Brendecke S, Assefa D, Helbing T, Frydrychowicz A, et al. Complex plaques in the proximal descending aorta: an underestimated embolic source of stroke. Stroke. 2010;41:1145-50
2. Harloff A, Hagenlocher P, Lodemann T, Hennemuth A, Weiller C, Hennig $J$, et al. Retrograde aortic blood flow as a mechanism of stroke: MR evaluation of the prevalence in a population-based study. Eur Radiol. 2019;29:5172-9.

3. Jarvis K, Soulat G, Scott M, Vali A, Pathrose A, Syed AA, et al. Investigation of aortic wall thickness, stiffness and flow reversal in patients with cyptogenic stroke: a 4D flow MRI study. J Magn Reson Imaging. 2020. https:// doi.org/10.1002/jmri.27345.

4. Hashimoto J, Ito S. Aortic stiffness determines diastolic blood flow reversal in the descending thoracic aorta: potential implication for retrograde embolic stroke in hypertension. Hypertension. 2013;62:542-9.

\section{Publisher's Note}

Springer Nature remains neutral with regard to jurisdictional claims in published maps and institutional affiliations.

\section{Submit your manuscript to a SpringerOpen ${ }^{\circ}$ journal and benefit from:}

- Convenient online submission

- Rigorous peer review

- Open access: articles freely available online

- High visibility within the field

- Retaining the copyright to your article

Submit your next manuscript at springeropen.com 